Ophthalmologe 2023 $\cdot 120: 72-75$

https://doi.org/10.1007/s00347-022-01597-4

Eingegangen: 7. Dezember 2021

Überarbeitet: 25. Januar 2022

Angenommen: 3. Februar 2022

Online publiziert: 8. März 2022

(c) Der/die Autor(en) 2022

\section{Therapieresistente noduläre Skleritis}

\author{
L. Hübner' (D) · C. Y. Mardin' · J. Held² · D. Tappe ${ }^{3}$. C. M. Hammer ${ }^{4}$ A. Bergua' \\ 'Universitätsaugenklinik Erlangen, FAU Erlangen-Nürnberg, Erlangen, Deutschland \\ ${ }^{2}$ Mikrobiologisches Institut, Klinische Mikrobiologie, Immunologie und Hygiene, Universitätsklinikum \\ Erlangen und Friedrich-Alexander-Universität (FAU), Erlangen-Nürnberg, Erlangen, Deutschland \\ ${ }^{3}$ Bernhard-Nocht-Institut für Tropenmedizin, Hamburg, Deutschland \\ ${ }^{4}$ Institut für Funktionelle und Klinische Anatomie, FAU Erlangen-Nürnberg, Erlangen, Deutschland
}

\section{Anamnese}

Eine 31-jährige Patientin stellte sich mit einer seit 3 Monaten bestehenden schmerzhaften Verdickung der bulbären Bindehaut nasal am linken Auge vor. Extern wurde bereits erfolglos lokal und systemisch mit Steroiden behandelt. Anamnestisch seien keine weiteren Augenerkrankungen bekannt; außer einem gut eingestellten Bluthochdruck und einer Hypothyreose sei sie allgemein gesund. Die aus Griechenland stammende Patientin berichtete über regelmäßige Heimatbesuche und den gelegentlichen Verzehr rohen Rindfleisches vom türkischen Metzger.

\section{Befunde}

In der ophthalmologischen Untersuchung zeigte sich eine noduläre Verdickung der bulbären Bindehaut nasal mit einer konjunktivalen und episkleralen Hyperämie (-Abb. 1a). Visus und Augeninnendruck waren unauffällig, Vorderkammer und Glaskörper reizfrei, der Fundus regelrecht. In der Ultraschallbiomikroskopie zeigte sich eine Verdickung der Bindehaut und Tenon mit einer darunter gelegenen nodulären Struktur ( $\bullet$ Abb. 1b). Beim genaueren Betrachten waren innerhalb der knotigen Formation längliche geschlängelte hyporeflektive Läsionen und Anschnitte hyperreflektiver zylindrischer Strukturen erkennbar (- Abb. 1c).

\section{Diagnose}

Es wurde die Verdachtsdiagnose eines Parasitenbefalls gestellt und eine operative
Sanierung angestrebt. Nach Inzision der nodulären Formation kamen mehrere Fadenwürmer im Bereich der Episklera und des Ansatzes des Musculus rectus medialis zum Vorschein, welche makroskopisch vollständig entfernt wurden (• Abb. 2a, b). In der molekularbiologischen Untersuchung mittels Polymerasekettenreaktion (PCR) konnte Nematoden-DNA nachgewiesen werden, die Sequenzierung erbrachte den Befund von Onchocerca lupi. Ein serologischer Suchtest auf eine Filarieninfektion blieb negativ, auch mikroskopisch zeigte sich kein Nachweis von Mikrofilarien im peripheren Blut. Ebenso blieben bildgebende Untersuchungen (Schädel-Magnetresonanztomographie, Abdomensonographie) ohne einen Hinweis auf weitere Organmanifestationen. In - Abb. 3 sind rasterelektronenmikroskopische Aufnahmen der geborgenen Nematoden dargestellt.

\section{Therapie}

Postoperativ wurde die Patientin systemisch antientzündlich (Prednisolon oral $100 \mathrm{mg}$ absteigend über 12 Tage) und lokal antibiotisch und antientzündlich mit Kombinationspräparaten (Augentropfen Gentamicinsulfat $5 \mathrm{mg} / \mathrm{ml}$ und Dexamethason $1 \mathrm{mg} / \mathrm{ml}$ 5-mal täglich, Augensalbe mit Gentamicinsulfat $5 \mathrm{mg} / \mathrm{g}$ und Dexamethason $0,3 \mathrm{mg} / \mathrm{g}$ zur Nacht) behandelt. Im Verlauf zeigte sich eine deutliche Verbesserung des Lokalbefundes (- Abb. 4). Auf eine Therapie mit einem Anthelminthikum wurde aufgrund des negativen Suchtestes für Filarieninfektionen im Blut verzichtet. 

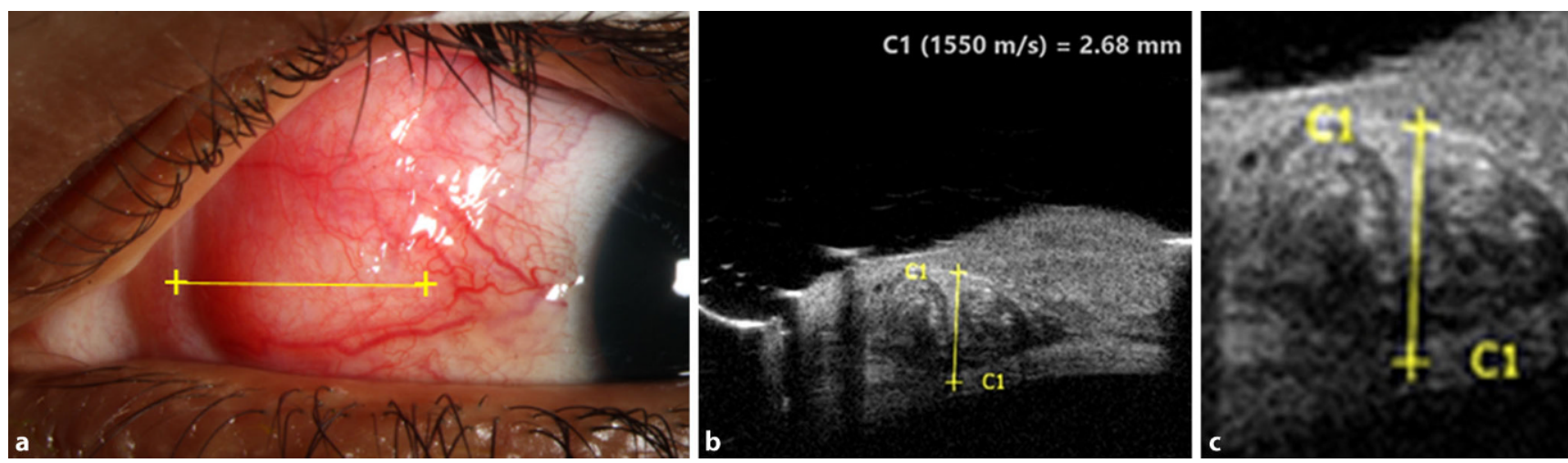

Abb. $1 \Delta$ a Klinischer Befund mit nodulärerVerdickung.b Korrespondierende Ultraschallbiomikroskopieaufnahme mit Verdickung der Bindehaut und darunter gelegener knotigen Struktur. c Nodulärer Prozess in stärkerer Vergrößerung mit den darin enthaltenen gewundenen hypo- und hyperreflektiven Strukturen
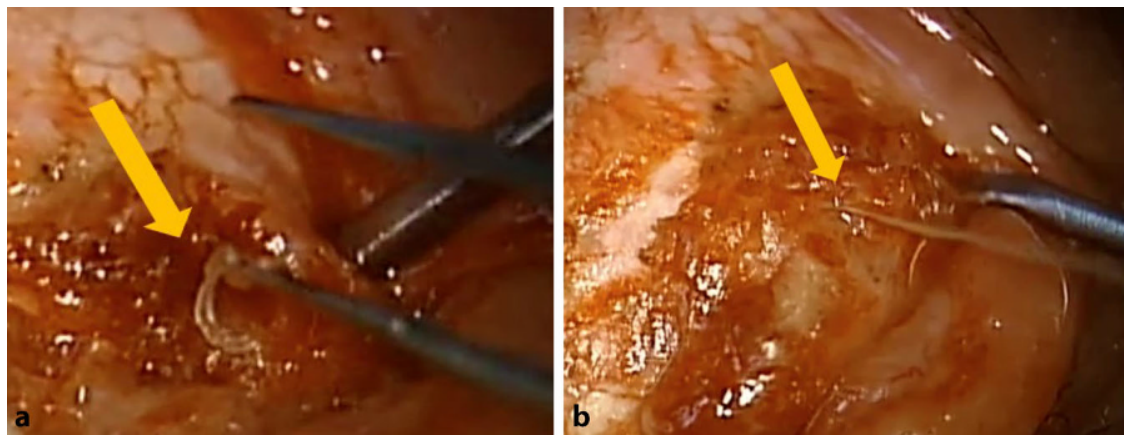

Abb. $2 \Delta$ Intraoperative Aufnahmen. a Größere Vergrößerung, b Übersichtsaufnahme. Mit gelbem Pfeil sind die farblos erscheinenden Nematoden gekennzeichnet

\section{Diskussion}

Bei Onchocerca lupi handelt es sich um einen Fadenwurm (Nematoden), welcher Hunde und seltener Katzen befallen kann [1]. Seit dem letzten Jahrzehnt gibt es Fallberichte über Infektionen bei Menschen aus der Türkei, dem Iran, Tunesien und zunehmend v. a. aus den USA [2]. Im Jahr 2014 wurde der erste und bislang einzige Fall aus Deutschland beim Menschen beschrieben, bei dem es sich jedoch höchstwahrscheinlich um eine aus der Türkei oder Tunesien importierte Infestation handelt [3]. Zuvor war eine Infektion bei einem deutschen Hund bereits 2002 veröffentlicht worden. Der Hund stammte aus einem Tierheim, die Ansteckungsquelle konnte nicht nachvollzogen werden [4].

Der genaue Lebenszyklus von O. lupi ist noch nicht vollständig verstanden. Bei infizierten Hunden entlassen gravide Weibchen Mikrofilarien in das subkutane Gewebe an verschiedenen Körperstellen. Während einer Blutmahlzeit werden die Mikrofilarien vom Zwischenwirt aufgenommen, welcher bislang nicht mit Sicherheit identifiziert wurde. Im Fokus steht besonders die Kriebelmücke, in Analogie zu anderen Onchocerca-Arten wie dem Erreger der Flussblindheit, O. volvulus [5].

Im Zwischenwirt entwickeln sich die Mikrofilarien zu Drittlarven (L3), wandern in das Stechwerkzeug und können während einer weiteren Blutmahlzeit auf den Endwirt übertragen werden. Auch die Inkubationszeit ist bislang nicht bekannt, basierend auf dem Wissen über andere Onchocerca-Arten wird sie auf mehrere Monate geschätzt [2]. Adulte Nematoden werden $4-12 \mathrm{~cm}$ lang und akkumulieren schließlich in gut perfundierten Geweben wie z. B. der Konjunktiva, der Episklera und dem Ansatz der geraden Augenmuskeln.

Bei Hunden können sich akute Infestationen durch periorbitale Schwellung, Lakrimation, Konjunktivitis, Protrusio bulbi, Chemosis, Photophobie, diffuses stromales Hornhautödem oder Netzhautablösungen äußern. Seltener finden sich schwere Verläufe mit Hornhautulzera, anteriorer und posteriorer Uveitis, Erblindung bis zur
Phthisis bulbi [2]. Darüber hinaus finden sich bei chronischen Verläufen am häufigsten multiple Knötchen (sub)konjunktival, retrobulbär oder auf der Nickhaut.

Beim Menschen verlaufen Infestationen mit $O$. lupi in der Regel harmlos und äußern sich in der Ausbildung bulbärer subkonjunktivaler Knötchen ohne einen Effekt auf den Visus [6]. Kürzlich wurde ein ähnlicher Fall mit der Beschreibung einer nodulären Skleritis publiziert [15]. Außerdem wurde eine extraokulare Beteiligung bei 3 Kindern unter 13 Jahren beschrieben. Hier fand sich eine spinale Manifestation in Form intraoder extraduraler Raumforderungen zwischen den Foramina intervertebralia von C2 und C4 [7-9].

Zur Diagnosesicherung ist die chirurgische Wurmextraktion nötig. Eine parasitologische Begutachtung kann die Würmer näher einordnen. Mittels PCR-Analyse aus dem gewonnenen Wurmmaterial kann O. lupi identifiziert werden $[3,10]$. Typische morphologische Charakteristika in der Lichtmikroskopie und Rasterelektronenmikroskopie sind in einer Übersichtsarbeit dargestellt [11]. In den meisten Fällen, außer solchen mit spinaler Beteiligung, wurden lediglich nichtgravide Weibchen gefunden. Dies lässt vermuten, dass der Mensch als Fehlwirt fungiert und die Immunreaktion erwachsener Patienten ggf. die Entwicklung des Nematoden stoppen kann.

Bislang gibt es keine evidenzbasierten Therapieprotokolle zur Behandlung einer O.-lupi-Infestation. Zunächst sollte eine komplette chirurgische Sanierung angestrebt werden, wie es in allen berichteten Fällen die Vorgehensweise war. Anthelmin- 

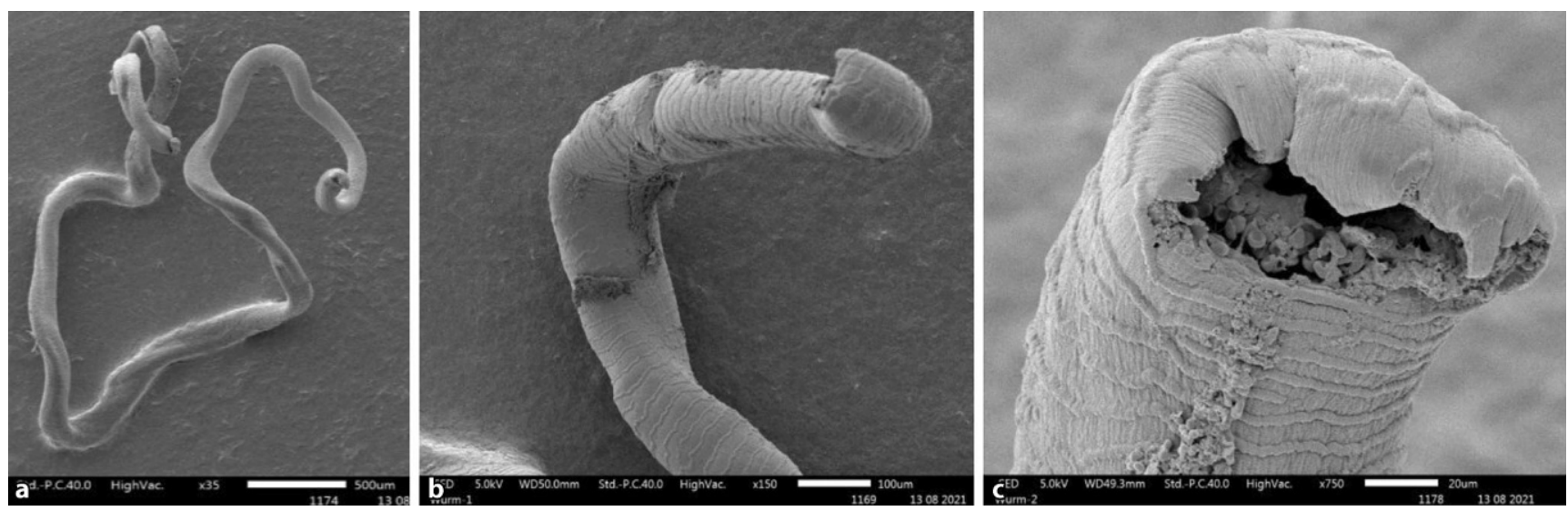

Abb. $3 \Delta$ Rasterelektronenmikroskopie-Aufnahmen. Die Fixierung der Präparate erfolgte mit einer phosphatgepufferten 2,5\%igen Glutaraldehyd-Lösung und $1 \%$ Osmiumtetroxid. Die Trocknung erfolgte mittels einer aufsteigenden Alkoholreihe und über Verdampfung von Hexamethyldisilazan (HDMS). Die Würmer wurden anschließend mit $20 \mathrm{~nm}$ Gold besputtert. a Vergrößerungsfaktor 35, b Vergrößerungsfaktor 150, c Vergrößerungsfaktor 750

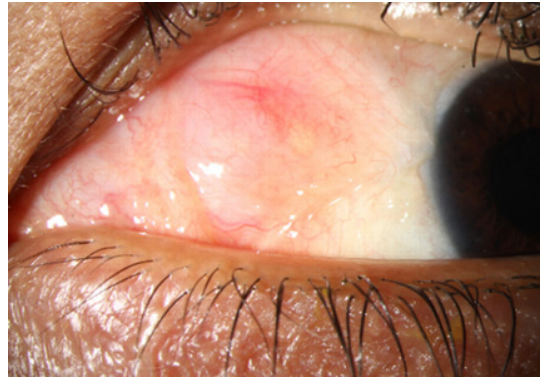

Abb. 4 A Klinischer Befund 4 Monate postoperativ

thika (v. a. Ivermectin) sollen Mikrofilarien abtöten und die Sterilisation der weiblichen adulten Würmer induzieren [12]. Der klinische Nutzen ist noch nicht vollständig geklärt. Da bei der hier vorgestellten Patientin ein negativer Suchtest für eine Filarieninfektion im Blut vorlag, wurde auf eine entsprechende systemische Therapie verzichtet. Die lokale und systemische Anwendung von Steroiden soll die Entzündungsreaktion nach Wurmextraktion reduzieren [13], wie sie auch bei dieser Patientin zu einer Verbesserung des Lokalbefundes führte. Gegebenenfalls können Antibiotika gegen das endosymbiontische Bakterium Wolbachia wirken [14].

\section{Fazit für die Praxis}

- Eine Ultraschallbiomikroskopie kann bei unklaren Raumforderungen der Episklera diagnostisch wichtige Hinweise liefern.

- O. lupi sollte in den Differenzialdiagnosen anderer parasitärer Augenerkrankungen beim Menschen bedacht werden, insbe- sondere wenn Aufenthalte in Risikogebieten bekannt sind.

- Bei Therapieresistenz nodulärer Skleritiden müssen neben den herkömmlichen immunologischen Diagnosen (wie z. B. Sarkoidose, Granulomatose mit Polyangiitis) auch seltene parasitäre Erkrankungen bedacht werden.

\section{Korrespondenzadresse}

\section{Hübner}

Universitätsaugenklinik Erlangen, FAU Erlangen-Nürnberg

Schwabachanlage 6, 91054 Erlangen,

Deutschland

lisa.huebner@uk-erlangen.de

Funding. Open Access funding enabled and organized by Projekt DEAL.

\section{Einhaltung ethischer Richtlinien}

Interessenkonflikt. L. Hübner, C.Y. Mardin, J. Held, D. Tappe, C.M. Hammer und A. Bergua geben an, dass kein Interessenkonflikt besteht.

Für diesen Beitrag wurden von den Autoren keine Studien an Menschen oder Tieren durchgeführt. Für die aufgeführten Studien gelten die jeweils dort angegebenen ethischen Richtlinien. Für Bildmaterial oder anderweitige Angaben innerhalb des Manuskripts, über die Patienten zu identifizieren sind, liegt von innen und/oder ihren gesetzlichen Vertretern eine schriftliche Einwilligung vor.

Open Access. Dieser Artikel wird unter der Creative Commons Namensnennung 4.0 International Lizenz veröffentlicht, welche die Nutzung, Vervielfältigung, Bearbeitung, Verbreitung und Wiedergabe in jeglichem Medium und Format erlaubt, sofern Sie den/die ursprünglichen Autor(en) und die Quelle ordnungsgemäß nennen, einen Link zur Creative Commons Lizenz beifügen und angeben, ob Änderungen vorgenommen wurden.

Die in diesem Artikel enthaltenen Bilder und sonstiges Drittmaterial unterliegen ebenfalls der genannten Creative Commons Lizenz, sofern sich aus der Abbildungslegende nichts anderes ergibt. Sofern das betreffende Material nicht unter der genannten Creative Commons Lizenz steht und die betreffende Handlung nicht nach gesetzlichen Vorschriften erlaubt ist, ist für die oben aufgeführten Weiterverwendungen des Materials die Einwilligung des jeweiligen Rechteinhabers einzuholen.

Weitere Details zur Lizenz entnehmen Sie bitte der Lizenzinformation auf http://creativecommons.org/ licenses/by/4.0/deed.de.

\section{Literatur}

1. Sréter T, Széll Z (2008) Onchocercosis: a newly recognized disease in dogs. Vet Parasitol 151:1-13

2. Rojas A, Morales-Calvo F, Salant H, Otranto D, Baneth G (2021) Zoonotic ocular Onchocercosis by Onchocerca lupi. Yale J Biol Med 94:331-341

3. Bergua A et al (2015) Human case of Onchocerca lupi infection, Germany, August 2014. Euro Surveill 20(16):pii=21099

4. Hermosilla C, Hetzel U, Bausch M, Grübl J, Bauer C (2005) First autochthonous case of canine ocular onchocercosis in Germany. Vet Rec 156:450-452

5. Otranto D et al (2012) Tracking the vector of Onchocerca lupi in a rural area of Greece. Emerg Infect Dis 18:1196-1200

6. Mowlavi G et al (2014) Human ocular onchocerciasis caused by Onchocerca lupi (Spirurida, Onchocercidae) in Iran. J Helminthol 88:250-255

7. Eberhard ML et al (2013) Zoonotic Onchocerca lupi infection in a 22-month-old child in Arizona: first report in the United States and a review of the literature. Am JTrop Med Hyg 88:601-605

8. Chen T et al (2015) Case report of an epidural cervical Onchocerca lupi infection in a 13-year-old boy. J Neurosurg Pediatr 16:217-221

9. Dudley RWR et al (2015) A cervical spine mass caused by Onchocerca lupi. Lancet 386:1372 
10. de Almeida M et al (2020) Duplex real-time PCR assay for clinical differentiation of Onchocerca lupi and Onchocerca volvulus. Am J Trop Med Hyg 103:1556-1562

11. Mutafchiev $Y$ et al (2013) Redescription of Onchocerca lupi (Spirurida: Onchocercidae) with histopathological observations. Parasit Vectors 6:309

12. Cantey PT et al (2016) The emergence of Zoonotic Onchocerca lupi infection in the United States-a case-series. Clin Infect Dis 62:778-783

13. Grácio AJS, Richter J, Komnenou AT, Grácio MA (2015) Onchocerciasis caused by Onchocerca lupi: an emerging zoonotic infection. Systematic review. Parasitol Res 114:2401-2413

14. Egyed Z et al (2002) Electron microscopic and molecular identification of Wolbachia endosymbionts from Onchocerca lupi: implications for therapy. Vet Parasitol 106:75-82

15. Hasanreisoglu Metal (2021) Case Report:A Human Case of Onchocerca lupi Mimicking Nodular Scleritis. Am J Trop Med Hyg 105(6):1782-1785

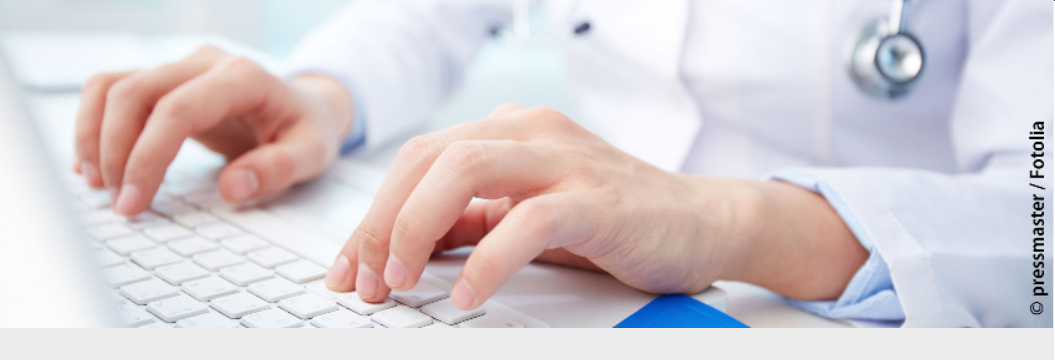

Für Autorinnen und Autoren: Ihr Beitrag in Die Ophthalmologie

Das sollten Sie bei Ihrer Einreichung beachten

Wir freuen uns über jede Einreichung in den Rubriken „Originalien“, „Übersichten“, "Kasuistiken" und „Bild und Fall".

Bitte beachten Sie bei der Erstellung Ihres Beitrags folgende Formatvorgaben:

\section{Originalien}

- Vorstellung und Diskussion von Methoden und Ergebnissen eigener Studien

- Gliederung: Hintergrund, Methoden, Ergebnisse, Diskussion

- max. 25.000 Zeichen inkl. Leerzeichen (Literatur, Tabellen und Abbildungslegenden bitte mitzählen)

- Kurze gegliederte Zusammenfassung (deutsch und englisch)

- 5 Schlüsselwörter (deutsch und englisch)

- Max. 6 Abbildungen inkl. Verweise im Text

- Max. 30 Literaturstellen

\section{Übersichten}

- Kritische Zusammenstellung und Diskussion relevanter wissenschaftlicher Ergebnisse

- max. 25.000 Zeichen inkl. Leerzeichen (Literatur, Tabellen und Abbildungslegenden bitte mitzählen)

- Kurze Zusammenfassung (deutsch und englisch)

- 5 Schlüsselwörter (deutsch und englisch)

- Max. 6 Abbildungen inkl. Verweise im Text

- Max. 30 Literaturstellen

\section{Kasuistiken}

- Kurzes Fallbeispiel, das Besonderheiten der klinischen Praxis, Fallstricke der Diagnostik und ungewöhnliche Krankheits- und Behandlungsverläufe aufzeigt

- Gliederung: Anamnese, Befund, Diagnose, Therapie und Verlauf, Diskussion

- max. 10.000 Zeichen inkl. Leerzeichen (Literatur, Tabellen und Abbildungslegenden bitte mitzählen)

- ohne Zusammenfassung und Schlüsselwörter

- Max. 6 Abbildungen inkl. Verweise im Text

- Max. 10 Literaturstellen

\section{Bild und Fall}

- Kurzes Fallquiz, bei dem der Leser miträtseln kann

- Gliederung: Teil 1 - Falldarstellung mit Anamnese, klinischem Befund und Diagnostik. Teil 2 - Diagnose, Therapie und Verlauf, ggf. weitere Hintergrundinformationen

- max. 10.000 Zeichen inkl. Leerzeichen (Literatur, Tabellen und Abbildungslegenden bitte mitzählen)

- ohne Zusammenfassung und Schlüsselwörter

- Max. 5 Abbildungen inkl. Verweise im Text

- Max. 5 Literaturstellen

Ausführliche Leitfäden finden Sie unter https://www.springer.com/journal/347 